Lints, T. (2010). The essentials of defining adaptation. In 4th Annual IEEE Systems Conference, pages 113-116. IEEE.

T. Lints, "The essentials of defining adaptation," in 4th Annual IEEE Systems Conference, pp. 113-116, IEEE, 2010.

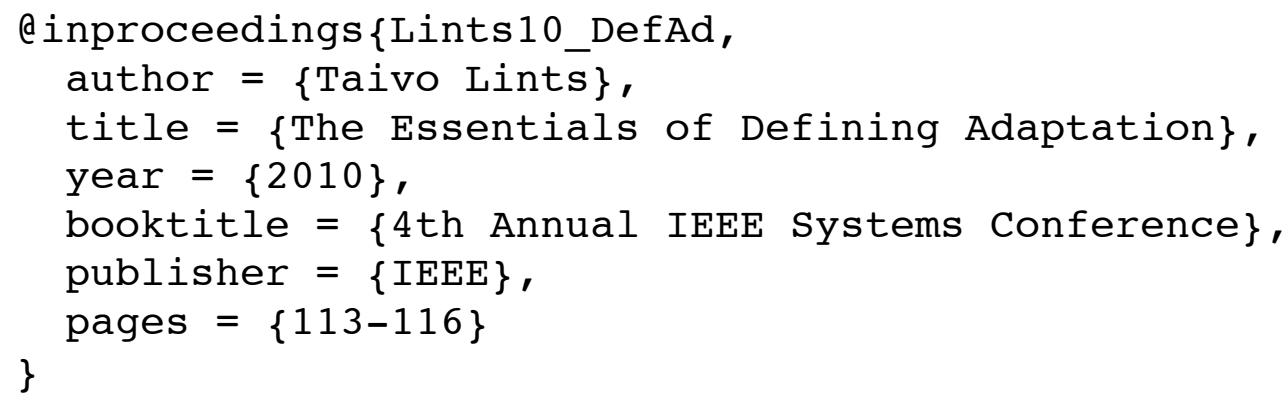




\title{
The Essentials of Defining Adaptation
}

\author{
Taivo Lints \\ Research Laboratory for Proactive Technologies \\ Tallinn University of Technology \\ Ehitajate tee 5, 19086 Tallinn, Estonia \\ Email: taivo@taivo.net
}

\begin{abstract}
Understanding the adaptivity of systems is among the key concerns in many areas of research and engineering. However, although widely used, the concepts of adaptation and adaptivity are fairly vague. This paper identifies and discusses the main types of adaptation definitions, and integrates the ideas from different fields of research into concise supportive guidelines that can make the adaptivity-related discourses more rigorous and more constructive, and thus facilitate the understanding, management and design of adaptive systems.
\end{abstract}

\section{INTRODUCTION}

The concept of adaptation has a considerable importance in many areas of research. Evolution theory is all about longterm adaptation, psychology is (among other things) interested in how people cope mentally with various changes in their life, business research tries to find ways to make companies survive and flourish in the ever more turbulent markets, armies rethink their strategies and become more flexible to be able to counter new threats, engineering disciplines move towards making systems better at reconfiguring and repairing themselves without costly and time-consuming human intervention, achieving sophisticated artificial life and artificial intelligence is in large part about finding out how to create systems that are highly adaptive and cope with the difficult and often unexpected situations they encounter, and so forth. When trying to understand, manage or create large complex systems, the issue of adaptivity almost always arises to the set of top concerns, even so much so that it has become quite common to use the term Complex Adaptive Systems when referring to the subject of research, management and design efforts in various fields that deal with systems consisting of large numbers of interacting components.

While adaptation is a widespread and frequently used concept, there exist a large number and high diversity of definitions of it that do not fully overlap, which can occasionally lead to unproductive confusion and misunderstandings. And most often the concept is used without any further explanations at all, just relying on its intuitive meaning in the common language, which, however, is fairly vague. In many cases such underspecified usage is indeed sufficient and fully acceptable, but sometimes, especially in engineering and scientific debates,

This work was supported in part by Research Laboratory for Proactive Technologies in Tallinn University of Technology, Department of Computer Control in Tallinn University of Technology, Estonian Doctoral School in ICT, Estonian Information Technology Foundation, and Estonian Ministry of Education and Research (grant SF0140113As08). a clearer and more rigorous approach would be preferred. In the following, a small sample of various definitions of adaptation and its various derivatives like adaptivity is given, together with highlighting some interesting and useful observations by various researchers, and then this broad spectrum of relevant ideas, many of which are not even included here due to limited space, is first summarized and evaluated, and then distilled into short guidelines on the essentials of defining adaptivity.

\section{A Small Selection of Definitions}

As adaptivity is an essential property of living systems, it is no wonder that biology as a discipline uses this concept extensively, and has been doing so already for a long time at least since Jean-Baptiste Lamarck's and Charles Darwin's publications in the 19th century. However, most likely due to the diversity and ubiquity of biological adaptational processes, there is no single universally accepted definition of adaptation even in biology. James P. Brock [1, page 3] points out that although the link with survivorship can be considered axiomatic, there are now at least seven classes of interpretation of adaptation: as function, as functional change, as change of form, as evolution, as specialization, as differential in fitness, and as absolute fitness. Brock, however, finds all these to be lacking in some aspect, and goes on to propose his own definition of adaptation as "performance of function in the context of interaction between an organism and its external environment such that survivorship is maintained between generations. From this definition, it follows that organisms possess only adaptive capacity and not adaptation, as such.”.

Toomas Tammaru [2], also elaborating mainly on the evolution theory's approach, considers an adaptation to be some state $A$, common to individuals of some population, that has appeared through natural selection to fulfill some specific function, and then puts forward an interesting line of thought. For the question "Is $A$ an adaptation?" to be scientifically answerable, Tammaru claims, at least two additional pieces of information must be provided. First, the function must be defined in relation to which adaptation is studied $-A$ may not be an adaptation in relation to function $H$ if it has evolved to fulfill function $F$, even if $A$ increases individual's fitness through rising the effectiveness of $H$ (he admits it creates complications in real analysis, as traits tend to be evolved to fulfill multiple functions to various degrees). Secondly, an earlier state must be defined to which $A$ is compared $-A$ 
could be an adaptation compared to some state $B$, but not an adaptation compared to some other state $C$.

Artificial Life researcher and philosopher Ezequiel Di Paolo, in his lecture slides [3], identifies another interesting factor that can affect adaptivity definitions. Namely, whether we consider a system to be adaptive may depend on the observer who chooses the scale and granularity of description. He gives an example: "Obstacle avoidance may count as adaptive behaviour if we describe navigation at a microscale where obstacles appear rarely in largely open and unobstructed segments of the environment. If the 'normal' environment is viewed at a macroscale as obstacle-rich, then avoidance becomes part of the "normal" behaviour rather than an adaptation.". In addition to spatial dimensions, Di Paolo has also discussed granularity in time [4]: "The adaptive event (or act) may be formed by the concatenation and parallel coordination of many other regulatory events, but there will be a point below which no further de-composition will be possible without losing the time-structure of the act. At that point what remains are raw processes. There is consequently a minimum temporal granularity in adaptivity."

Human as an advanced lifeform (in the context of Earth biosphere) displays a wide variety of adaptive behaviors, and thus most of the research fields that study humans inevitably also deal with adaptivity to some degree. In the context of defining flexibility, rigidity and adaptation in psychology, William A. Scott points out that adaptation is generally a multilateral process [5]: "Broadly conceived, adaptation implies two kinds of matching: a matching between the requirements of an organism and the resources of its environment, and also a matching between the requirements of an environment and the resources of its inhabitants. Both organism and environment are presumably subject to some degree of modification through action of the other.". He also emphasizes that "'adaptation' implies a particular frame of reference concerning the person and his environment. A given behavior may be judged adaptive with reference to one environment and not with respect to another. Adaptation to an immediate situation may impede adaptation to a situation that is subsequently encountered. Similarly, a given behavior may be judged adaptive from the standpoint of one aspect of the person, but not with respect to another aspect.". Another psychologist, O. J. Harvey, has the following opinion [6]: "In the ultimate sense, adaptability means the capacity to behave in ways maximally consonant with the attainment of ends or goals. Adaptable behavior thus becomes synonymous with appropriate behavior, 'appropriate' defined as the degree to which a particular act facilitates or runs counter to the attainment of a sought end. Obviously behavior which is consonant with one end may be, and frequently is, incompatible with other, simultaneously extant, goals.". Ployhart \& Bliese [7], on the other hand, propose that "Individual adaptability represents an individual's ability, skill, disposition, willingness, and/or motivation, to change or fit different task, social, and environmental features.", and Grisogono \& Ryan [8] take the following approach: "We use the term adaptation to encompass all the ways in which complex adaptive systems are able to improve their 'fitness' or success in their environment, through continual variations and fitness-linked selection operating on the systems to eliminate those variations that decrease success. This includes both evolutionary processes and learning processes, as well as various hybrid forms such as human design and creativity, and the evolution of culture.”. The latter is quite a widespread view on adaptation - the evolutionary process of biological systems generalized to all domains - which was proposed (or at least popularized) by John H. Holland in the 1960s and 70s. Another perspective on Holland's viewpoint is provided by Melanie Mitchell [9, page 92], in the context of genetic algorithms, as "the rough maximization of on-line performance is what goes on in adaptive systems of all kinds, and in some sense this is how 'adaptation' is defined".

Several quantitative measures of adaptivity have been proposed, too. Evolution theory has provided a well-defined measure of adaptation (mostly in the sense of adaptedness) - the adaptive value of a genotype is measured as the reproductive success of individuals with that genotype. However, as pointed out by Kalevi Kull [10], the initial notion of harmony between an organism and the environment has been partially left behind here, and Emilio F. Moran [11, page 10] adds that the adequacy of reproductive success as an index of (human) adaptation is made questionable by the problem of human overpopulation. Michael Conrad [12] defines an "extreme measure" of adaptability of a given biota as the most uncertain environment in which it is capable of remaining alive indefinitely, and quantifies it via entropies, whereas Lotfi A. Zadeh [13] proposes that (roughly speaking) a system is adaptive with respect to a set of operating conditions and a class of performance values if its performance in those conditions stays within that class, and the adaptivity of two systems could be in some cases weighed against each other by comparing the sets of acceptable operating conditions. However, Conrad's and Zadeh's definitions do not care if the survival or required performance is achieved with the help of dynamic adjustment processes or just by having enough durability or appropriate functionality already from the very beginning, thus these definitions do not differentiate between adaptivity and robustness. Finally, Anne-Marie Grisogono [14] describes interesting possibilities to quantify the success of adaptation by measuring: the speed with which the system can 'move' on its own fitness landscape and replace capabilities of lower or declining fitness with new ones better suited to creating success in its context; the ability to stabilize and protect useful properties; and the ability to modify its environment to maintain or increase its local habitability, or to foster the emergence of habitable regions elsewhere.

\section{DISCUSSION}

It seems to be possible to identify the main sources of ideas that have generated or inspired a large part of the definitions: evolution theory, cybernetics and control theory, and studies of human behavior both on the level of everyday experience and in scientific disciplines. They have each some assumptions 
and biases which may be adequate within their own domains but should be explicitly taken into account in the process of interdisciplinary generalization.

The evolution theory approach considers adaptation to be a process where variations of existing individuals are being generated and where selection operates on those variants, eliminating the less fit ones. Such an idea can be taken as a generalization of many adaptational processes, both in biology and elsewhere. It emphasizes the importance of feedback (through selection) in adaptation, and provides the notion of fitness, which in a more general setting would be a measure of how well the system is performing with regard to its goals. The latter leads to the concept of fitness function, which in turn provides us with a very helpful mental tool in the form of adaptive landscape. In addition, adaptation as viewed through the lens of evolution theory carries with it the suggestion for open-endedness, i.e., the system is expected to be able to keep improving itself and generating novelties.

Although this might sound as a great approach generalizable to all kinds of adaptational processes, one still has to exercise some caution in doing so. First of all, adaptational behavior can also be displayed by a system that does not use the variation-selection loop but instead is able to accurately enough estimate the required states and actions and generate them in "one shot". Surely, such a system might not necessarily be particularly adaptive in the long run because of the lack of learning from experience, but by no means does this automatically lead to considering the system incapable of any adaptive action. Secondly, the evolution theory approach tends to guide the thought to the selection being mostly an external force, which in a more general setting might not always be the best mental model. And thirdly, as the concept of teleology is generally treated with high suspicion when discussing biological evolution, the definitions of adaptation stemming from evolution theory tend to lack the explicit consideration of goals of the adapting system.

The definitions deriving from cybernetics and control theory consider the essence of adaptation to be coping with perturbances and keeping main system parameters near some setpoint. And indeed, a lot of adaptation all around and inside us certainly is concerned with homeostasis: keeping the system in some state in spite of the various perturbations that try to push it away. This approach leads to helping understanding adaptational processes with the mental tools of state-space and attractors in it. A strong emphasis here is on the notion of feedback - a concept that both was popularized largely by cybernetics and also forms one of the main supportive pillars of that field.

While in principle the target states and setpoints could be defined with enough generality or abstractness to allow the cybernetics and control theory approach to cover basically all sorts of adaptation, in practice there still remains the problem of leading the thought towards closedness, as opposed to evolutionary approach's hinting on long-term open-ended development. Radical changes of the system are usually not considered acceptable in this way of thinking. There tends to be an expectation that after a successful adaptation process the system has returned, in large part, to its pre-perturbation state. Also, as the main focus is in keeping the system near some setpoint, by whatever means available, the cybernetics and control theory inspired adaptation definitions sometimes do not care much about whether there is an active process behind it or just passive resistance, and thus mix adaptivity and robustness.

The understanding and interpretation of the concept of adaptation in common everyday language is likely to be based mostly on human behavior. It is a bit difficult to provide a good summarization of this informal usage of the word, but the core seems to be some change in a system that helps it to cope and I or succeed in or after situations of changing circumstances. As opposed to the previously described cybernetics and control theory approach, in everyday language the distinction between the ability to adapt and the ability to withstand disturbances without (inner) change is typically quite clear.

The common usage of the concept of adaptation has a considerable effect on how it is used in various fields of scientific enquiry, providing a kind of a background feeling of which approaches might be acceptable and which should be treated with suspicion due to conflicting with the common sense. However, the common usage is fairly vague and underspecified, does not usually ponder on the importance of feedback and goals, and is of the type "I know it when I see it" rather than something easily conveyable.

Various more scientific approaches to human studies like psychology and organizational research have in principle the same observers-investigators and the main targets of interest as the common language case, i.e., humans being interested in humans, and correspondingly these fields have a noticeable overlap with everyday language usage, but they surely try to be less vague and more detailed in their definitions. Thanks to the humans being widely regarded as systems that can really set goals for themselves and who have, at least at a suitable level of abstraction, some degree of proactivity, the human studies' approach to understanding and defining adaptation at times explicitly pays closer attention to the role of goals, and to the possibility of having several conflicting and / or changing goals, doing it from a slightly different perspective than the fitness function based approaches.

On the other hand, partly due to the complexity of human behavior, many psychological definitions of adaptation and adaptivity still remain quite vague. On top of that there is another problem stemming from the effort of achieving more clarity in an inherently complex topic - while various quite detailed definitions do have been put forward over time, they tend to be rather diverse and are not yet converging towards forming a widely accepted psychological definition of adaptivity.

\section{The Essentials of Defining Adaptation}

In general, adaptation as a process is apparently about changing something (itself, others, the environment) so that it would be more suitable or fit for some purpose than it would 
have otherwise been. This includes reacting to disturbances by lessening their negative impact and, if possible, by restoring the pre-perturbation fitness levels, as well as improving the system and / or situation in an otherwise stable environment.

In biological systems, the most common (but typically implicit) goal is survival, and the mechanisms of adaptation are evolution on the longer timescale and developmental, physiological, behavioral and learning processes on the level of individual organisms. Survival, however, is not an universal goal even in biological systems. Continuation of the species may sometimes be better served by having individuals' lifetimes less than maximum possible, in which case single organism's target is not the maximization of its own survival anymore. In advanced lifeforms who are deliberately setting their aims, some individuals may even decide to care neither about remaining alive itself for a long time nor about helping the species to go on. For lifeforms with less mental capacity similar "decision" can result from malfunctioning of evolved survival mechanisms. Such organisms are not necessarily less adaptive: they may well be enjoying their life more and from the viewpoint of maximizing the joy be performing remarkably well. Further, in artificial systems it is typically the system designer who sets the goals and fitness functions, and survival is hardly ever the topmost purpose of an artifact. Surely, more advanced artificial systems that gain enough autonomy can become less distinguishable from biological systems in the process of goal setting, but some of the aims they develop may well be even less similar to those of most biological systems than the aims of today's artifacts.

This leads to the relativity of adaptation: there is a need to specify the frames of reference. For a statement "this system is adaptive" to have any rigor it should be complemented with some specifications. First of all, it is important to point out the goal of the system with regard to which it is considered to behave adaptively. As noted, in biology the goal is typically implicitly taken to be survival, but it is not an universal aim of systems. Also, the system may have multiple simultaneous, and possibly conflicting, goals. Secondly, the environment should be described in which the system can be said to behave adaptively (because no real system can do universally well in all possible conditions, at least with regard to any practically feasible goal). Thirdly, time frames are significant - what is the time interval in which the system performs well with regard to its aims (as, for example, some shortsighted fitness-improving actions can lead to later significant losses). And fourthly, not all of the processes in the system under study are necessarily beneficial for the specified goal, thus instead of only speaking about the adaptivity of the system in general, a more detailed description may sometimes be appropriate (where some of the system's processes are considered adaptive and others not). So in conclusion (and in the spirit of Lotfi A. Zadeh [13]): due to the relativity of adaptation it does not really matter whether a system is adaptive or not (they almost all are, in some way or another), but with respect to what it is adaptive.

\section{ACKNOWLEDGMENT}

Thanks to Leo Mõtus for providing me the possibility to do highly unconstrained research.

\section{REFERENCES}

[1] J. P. Brock, The Evolution of Adaptive Systems: The General Theory of Evolution. Academic Press, 2000.

[2] T. Tammaru, "Kohastumuse definitsioonist ja selle rakendamisest," in Adaptatsiooni teooria. Schola Biotheoretica XXVI, I. Puura, Ed. OÜ Sulemees, 2000, pp. 17-21, in Estonian.

[3] E. A. Di Paolo, "Spring 2006 adaptive systems course slides," http: //www.informatics.sussex.ac.uk/users/ezequiel/teaching.html, 2006.

[4] — "Autopoiesis, adaptivity, teleology, agency," Phenomenology and the Cognitive Sciences, vol. 4, pp. 429-452, 2005.

[5] W. A. Scott, "Flexibility, rigidity, and adaptation: Toward clarification of concepts," in Experience, Structure \& Adaptability, O. J. Harvey, Ed. Springer, 1966, pp. 369-400.

[6] O. J. Harvey, "Ends, means, and adaptability," in Experience, Structure \& Adaptability, O. J. Harvey, Ed. Springer, 1966, pp. 3-12.

[7] R. E. Ployhart and P. D. Bliese, "Individual adaptability (I-ADAPT) theory: Conceptualizing the antecedents, consequences, and measurement of individual differences in adaptability," in Understanding Adaptability, A Prerequisite for Effective Performance within Complex Environments, C. S. Burke, L. G. Pierce, and E. Salas, Eds. Elsevier, 2006, pp. 3-39.

[8] A.-M. Grisogono and A. Ryan, "Operationalising adaptive campaigning," in International Command and Control Research and Technology Symposium, 12th ICCRTS, 2007.

[9] M. Mitchell, An Introduction to Genetic Algorithms. Cambridge, MA, USA: MIT Press, 1998.

[10] K. Kull, "Adaptatsioon: funktsioon, tähendus või järglaste arv?" in Adaptatsiooni teooria. Schola Biotheoretica XXVI, I. Puura, Ed. OÜ Sulemees, 2000, pp. 9-10, in Estonian.

[11] E. F. Moran, Human Adaptability: An Introduction to Ecological Anthropology, 2nd ed. Westview Press, 2000.

[12] M. Conrad, Adaptability. The Significance of Variability from Molecule to Ecosystem. Plenum Press, 1983.

[13] L. A. Zadeh, "On the definition of adaptivity," Proceedings of the IEEE, vol. 51, no. 3, pp. 469-470, 1963.

[14] A.-M. Grisogono, "Success and failure in adaptation," in Proceedings of the Sixth International Conference on Complex Systems, A. Minai, D. Braha, and Y. Bar-Yam, Eds. New England Complex Systems Institute, 2006. 